\title{
Preliminary experimental tests of local erosion at river piers with a particular shape
}

\author{
L. Ciaravino \& G. Ciaravino \\ Department of Hydraulic, Geotechnical and Environmental Engineering, \\ University of Naples Federico II, Italy
}

\begin{abstract}
In the present study early experimental tests concerning local erosion phenomena have been carried out on a particular shape of the already effective circular piers in order to determine if any improvement is verified in their shape coefficient (with a corresponding decrease in scour). In particular, the circular pier foundation has been shaped as a truncated cone, partially or totally sunk into the mobile bed. In the experimental tests a preliminary comparison has been effected between scour at uniform circular pier and non-uniform piers with two different values of bevel angle $\beta$ for the truncated cone foundation. Experimental results have shown that, for both values of $\beta$, the scour depth has been lower than that identified in experimental tests conducted on circular piers of the same diameter but without the truncated cone foundation. This interesting results open up an encouraging research field.
\end{abstract}

Keywords: local erosion, river piers, shape effect, experimental test.

\section{Introduction}

Erosion in riverbeds and banks, also for reaches with considerable length, is often caused by isolated man-made structures. Such circumstance is frequently verified when in the river body bridge abutments or piers are built. In the case of piers, for example, it has been observed that local erosion is specifically affected by shape and size of the pier as well as by its position with respect to stream flow [1-4]. For purposes of application, in case of scour $Y_{s}$ corresponding to an isolated pier, considerable acclaim was paid to the equation suggested by Breusers et al. [4] which is effectively an envelope equation of the various experimental results: 


$$
\frac{Y_{s}}{b_{p}}=\left[2.0 \tanh \left(\frac{y}{b_{p}}\right)\right] \cdot f_{1}\left(\frac{V}{V_{c s}}\right) \cdot f_{2}(\text { shape }) \cdot f_{3}\left(\alpha, \frac{L_{p}}{b_{p}}\right)
$$

where $b_{p}$ and $L_{p}$ are pier width and pier length respectively, $y$ is the approach flow depth, $V$ and $V_{c s}$ are the average approach velocity and the critical entrainment velocity and $\alpha$ is the angle relative to approach flow direction. In eqn. (1) $f_{l}$, depended to the $V / V_{c s}$ ratio, can be determined from the $V_{c s}$ value defined trough the following equation by Hanco [5]:

$$
V_{c s}=1.2 \cdot \sqrt{g \cdot d_{s} \cdot \frac{\rho_{s}-\rho}{\rho}} \cdot\left(\frac{y}{d_{s}}\right)^{0.2}
$$

where $\rho, \rho_{S}$ and $d_{S}$ are fluid density, sediment density and sediment size, respectively; furthermore $f_{3}$ can be deduced from the well-known nomograph set up by Laursen and Toch [2] as a function of the angle $\alpha$ and the $L_{p} / b_{p}$ ratio; finally $f_{2}$ is a coefficient depended to the pier shape. Hence, the search for pier geometric conformations, which provide favourable values of the $f_{3}$ and $f_{2}$ coefficients, is of considerable importance in the field of pier design. In technical practice, designers frequently opt for the uniform circular pier which not only has a favourable value of shape coefficient but also maintains this value for any variation in stream flow direction.

In this study, experimental scour tests have been carried out for a particular shape of the already effective circular piers in order to ascertain any improvement in their shape coefficient (through a decrease in scour).

\section{Experimental set-up}

It is well known that the lower part of a river bridge pier (hereafter called the pier base) is subject to the action of the stream flow and of the sediment transported in the proximity of the river bed and in suspension. Therefore it seems fitting to protect the pier (and any reinforcing elements) from this action on its surface.

The abrasive action of transported sediment is a major issue for piers located in rivers subject to torrential flows where the material transported substantially comprises sharp-edged stones. Typically, in order to distribute stresses in the foundation and also to protect the pier base, the pier is configured in one of several more or less massive shapes that lead to considerable scour on the mobile riverbed $[6,7]$. Taking these considerations into account, it was hypothesised that the circular pier could be given a truncated cone shaped foundation partially or totally sunk into the erodible bed, as shown in Figure 1.

In fact Figure 1 shows the geometries tested in the experimental installation corresponding to six circular piers of diameter D varying from $0.08 \mathrm{~m}$ to $0.20 \mathrm{~m}$ $(0.080 ; 0.100 ; 0.125 ; 0.140 ; 0.160 ; 0.200)$ with and without a truncated cone base as their foundation. Two truncated cone bases have been tested, with the bevel angle $\beta$ relative to the vertical axis equal to $30^{\circ}$ and $45^{\circ}$ and a greater 
Piers with diameter $\mathrm{D}$ from 0.08 to $0.20 \mathrm{~m}$

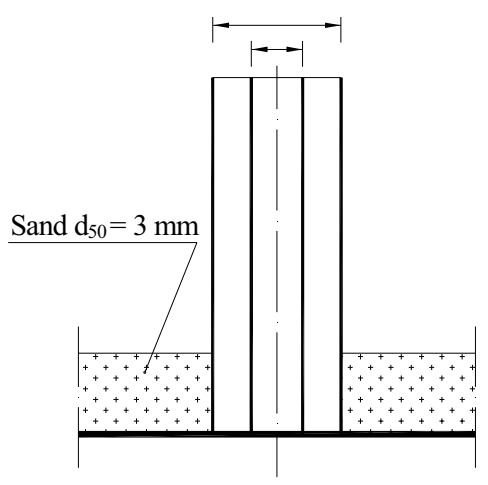

Piers with diameter $\mathrm{D}$ from 0.08 to $0.16 \mathrm{~m}$

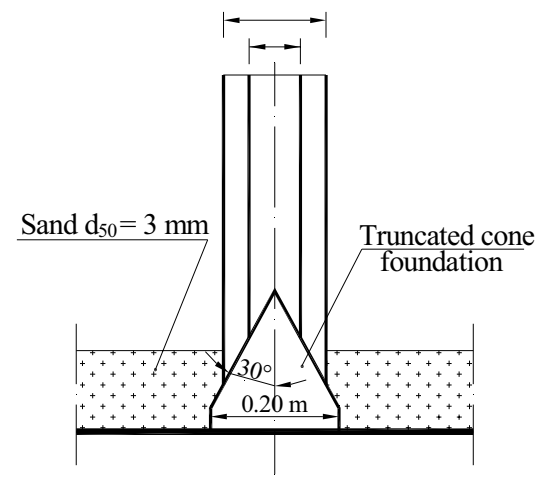

Piers with diameter D from 0.08 to $0.20 \mathrm{~m}$

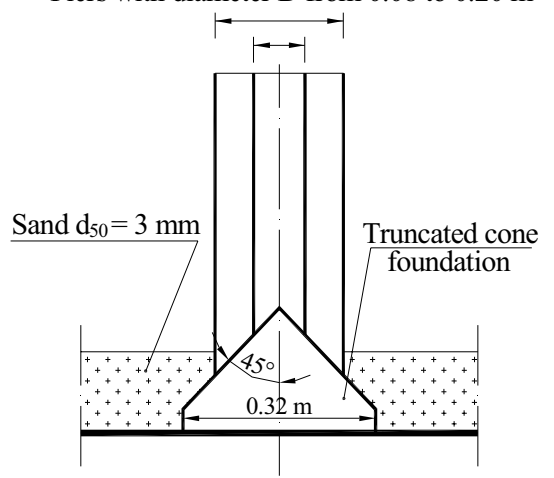

Figure 1: Circular piers with or without truncated cone base used in the tests. 
diameter respectively equal to $0.20 \mathrm{~m}$ and $0.32 \mathrm{~m}$ (Figure 1). It was decided to restrict the value of the $\beta$ angle in order to ensure geometries covering a smaller surface area, which is an important technical consideration. As the circular pier with a diameter of $0.20 \mathrm{~m}$ almost entirely covers the truncated cone base with angle $\beta=30^{\circ}$, a total of seventeen geometries have been tested (six piers without the truncated cone base, six piers with a truncated cone base with angle $\beta=45^{\circ}$ and five piers with a truncated cone with angle $\beta=30^{\circ}$ ) which enabled an initial comparison of the behaviour of the uniform circular pier (whose foundation is hypothesized at a level deeper than the expected scour) and that of the shaped pier with the truncated cone base. The set-up for the experimental tests was housed in a variable slope channel (maximum obtainable slope $18^{\circ}$ ) measuring $27.26 \mathrm{~m}$ in length with a closed circuit supply currently in use in the Experimental Laboratory at the Department of Hydraulic of the University Federico II of Naples. In particular, the experimental set-up comprises:

- $\quad$ an initial ascending section measuring $3.00 \mathrm{~m}$ in length;

- $\quad$ a section $2.40 \mathrm{~m}$ in length reproducing a mobile riverbed (in which the piers being tested have been sunk). The bed erodible material is made up of variable grain-size sand passed through a series of sieves which make it possible to reconstruct the granulometric curve that gives a median sediment size $\mathrm{d}_{50}$ of $0.003 \mathrm{~m}$;

a return slide measuring $2.10 \mathrm{~m}$ in length;

a device for trapping the material transported by the stream, which is constituted by a grill of suitable mesh to ensure that this material does not end up in the recycle circuit;

a weir for measurement and control positioned at the end of the channel (which also completes the work of the entrapment device).

In the preliminary tests carried out, the slope of the mobile river bed has been made equal to $1 \%$, while the test flow rate $\mathrm{Q}$, equal to $0.0118 \mathrm{~m} 3 / \mathrm{s}$, has been determined (by a series of preliminary tests) as the flow rate that, with the assigned slope, determines conditions close to that of incipient motion of the sediment reproducing the mobile river bed (in clear-water conditions). The flow rate is measured by two devices (one of which is electromagnetic) sending their readings to a control desk which also regulates the slope of the channel. In such experimental conditions low values of water depths and scour depths have been obtained; as a consequence the preliminary tests have pointed out that, already with a test duration of $1.5 \mathrm{~h}$, the solid transport with consequent increase of erodible material found in the trapping device can be considered negligible.

This finding is consistent with the well known circumstance that the time needed to develop equilibrium depths of scour at bridge piers is approximately proportional to the volume of the equilibrium scour hole for a given flow condition $[8,9]$.

\section{Experimental tests}

As already noted, the experimental tests have been carried out in order to compare the behaviour of the various configurations (reported in Figure 1) with 
respect to the expected scour phenomena around the piers, rather than achieve an absolute determination of scour entity. In this phase of research, therefore, the tests have been effected for a single value of slope, flow rate and limited test duration, as already mentioned in the previous paragraph, and thus in a single framework of hydrodynamic comparison. Tests have been carried out for the seventeen described geometries (see Figure 1) and the local erosion measurements taken at the end of the tests have been collected and expressed in graphs and tables (not reported here for brevity's sake). In particular, the tables report the scour values for the horseshoe-vortex and the local erosion measurements taken downstream of the pier corresponding to the wake-vortex (which occur in much of the reproduced downstream river bed). The tests have shown that scour in the area immediately surrounding the pier has the typical "horseshoe" shape whose front width (at bed level) $\mathrm{W}_{\mathrm{S}}$, corresponding to an arc of $180^{\circ}$, has been almost constant in size; in particular, it has been noticed that:

- $\quad$ in the case of piers with a truncated cone foundation, $\mathrm{W}_{\mathrm{S}}$ appears to depend substantially on the angle assigned to the truncated cone base and is, therefore, practically constant as the pier diameter $\mathrm{D}$ varies for each of the two bases tested (in fact the tests show that the base with $\beta=30^{\circ}$ has $\mathrm{W}_{\mathrm{S}}$ values that are on average about $25 \%$ smaller);

where no truncated cone foundation is present, $\mathrm{W}_{\mathrm{S}}$ is variable and increases with the pier diameter $\mathrm{D}$ (with values substantially ranging between the values measured for the base with $\beta=30^{\circ}$ and the values measured for the base with $\beta=45^{\circ}$ ).

The described characteristics can be deduced from the graph in Figure 2.

The experimental data collected made it possible to extrapolate (at the end of each test) the scour values of the area in front of the pier and, therefore, relative to the horseshoe-vortex: such values are reported in Table 1.

As can be observed in Table 1, the scour depth $Y_{S}$ (starting from a practically common value corresponding to the smallest diameter $\mathrm{D}=0.080 \mathrm{~m}$ ) medially increases as pier diameter $\mathrm{D}$ increases.

Furthermore, in the tests conducted on piers with a truncated cone base (both those with $\beta=30^{\circ}$ and those with $\left.\beta=45^{\circ}\right), Y_{S}$ has a lower value for equal diameters (again excluding the common value corresponding to the smallest diameter $\mathrm{D}=0.080 \mathrm{~m}$ ) than in the tests conducted on piers without the truncated cone foundation. In other words, in the experimental tests with piers on a truncated cone base, for every single dimension $\mathrm{D}$, scour values show a very significant percentage reduction. In particular, the reduction in scour is greater for the base with $\beta=45^{\circ}$.

Moreover, the percentage variation follows a medially decreasing pattern as the diameter D increases. Basically, the experimental tests show that, with all other conditions being equal, the truncated cone foundation has a more favourable shape coefficient.

In the examined cases, by assigning the uniform circular pier a shape coefficient equal to 1 (as suggested when using Breusers et al.'s eqn. (1)) [4] and attributing the entire reduction of the scour depth to the shape coefficient, shape coefficients would be obtained which can be estimated, for scour purposes, to be 


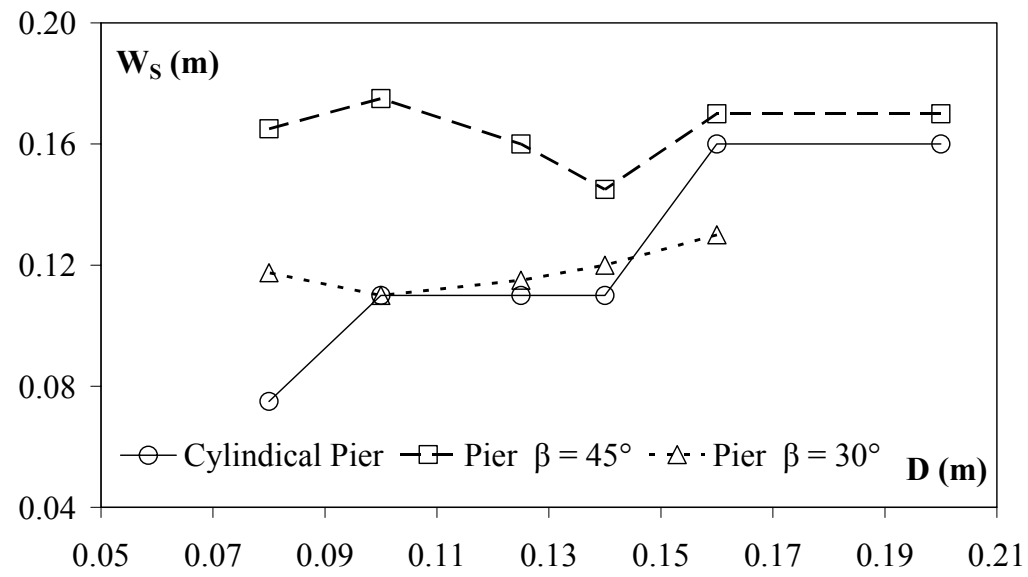

Figure 2: $\quad$ Front scour width $\mathrm{W}_{\mathrm{S}}$ versus $\mathrm{D}$ (maximum experimental values).

Table 1: Experimental values of scour corresponding to the horseshoevortex.

\begin{tabular}{cccc}
\hline & \multicolumn{3}{c}{$Y_{S}(\mathrm{~m})$} \\
\cline { 2 - 4 } $\mathrm{D}(\mathrm{m})$ & uniform pier & $\beta=30^{\circ}$ & $\beta=45^{\circ}$ \\
\hline 0.080 & 0.060 & 0.062 & 0.060 \\
0.100 & 0.095 & 0.061 & 0.057 \\
0.125 & 0.107 & 0.073 & 0.058 \\
0.140 & 0.089 & 0.064 & 0.062 \\
0.160 & 0.119 & 0.086 & 0.068 \\
0.200 & 0.112 & --- & 0.076 \\
\hline
\end{tabular}

on average between 0.70 and 0.75 . These values are extremely favourable shape coefficients that can be compared to the ones currently assigned to best configurations of river piers.

It should be remembered that (again in the use of eqn. (1)), considering piers with the sophisticated elliptical cross section with the major axis parallel to the direction of stream flow, a shape coefficient of almost 0.75 can be adopted.

Moreover, it is also known that should the stream not run parallel to the major axis of such an elliptical pier, then scour would increase considerably according to the angle relative to approach flow direction $\alpha$ : as can be deduced from eqn. (1) and from the above mentioned nomograph of Laursen and Toch which makes it possible to deduce the coefficient $f_{3}[2,4]$.

This increase in scour as the angle relative to approach flow direction varies is obviously not expected for circular piers and, therefore, for the piers with a truncated cone base used in the experimental tests presented here which are axially symmetrical. 
Moreover, as shown by Melville and Raudkivi [9], in case of circular piers a reduction in scour depth can be achieved if the top of the foundation is within the scour hole but below the bed surface otherwise an increase in scour depth could be verified.

In a mobile bed the foundation position with reference to the bed surface can vary with time so that the evaluation of scour depth appears to be uncertain. Such a problem seems to be less important in case of piers with truncated cone foundation whose width gradually varies with the level considered.

Therefore a considerable technical advantage can be altogether expected in using truncated cone foundations like those tested which are certainly deserving of further investigation from both a quantitative and a qualitative point of view.

\section{Discussion on experimental results}

In order to analyse the described experimental results with a view to their application, Figure 3 reports the experimental values of the $Y_{S} / b_{p}$ and $y / b_{p}$ ratios (with $b_{p}=\mathrm{D}$ ) so as to obtain a comparison with some empirical equations whose representation can be attributed to these dimensionless ratios.

In particular, the comparison has been made using the eqn. (1) proposed by Breusers et al. [4] and the more recent equation proposed in the Bridge Scour Manual of the Florida Department of Transportation [7] in the simple case of a single pier.

The latter equation, developed substantially by Sheppard [6] on the basis of experimental data (Sheppard is author of several papers here not mentioned for brevity's sake), for the clearwater field tested here $\left(0.47<V / V_{c s}<1\right)$, can be written as follows:

$$
\frac{Y_{S}}{b_{p}^{*}}=2.5 \tanh \left(\frac{y}{b_{p}^{*}}\right)^{0.4}\left\{1-1.75\left[\ln \left(\frac{V}{V_{c s}}\right)\right]^{2}\right\} \frac{\frac{b_{p}^{*}}{d_{50}}}{0.4\left(\frac{b_{p}^{*}}{d_{50}}\right)^{1.2}+10.6\left(\frac{b_{p}^{*}}{d_{50}}\right)^{-0.13}} .
$$

In eqn. (3), in addition to the already mentioned symbols, $b_{p} *$ indicates the socalled effective diameter (which in the case of uniform cylindrical piers coincides with the pier diameter D) while for the critical velocity $V_{c s}$ (taking into account Shield's diagram) the following equation is suggested:

$$
V_{c s}=2.5 \cdot U_{*_{c}} \ln \left(\frac{y}{2.72 \cdot Z_{0}}\right) .
$$

In eqn. (4) (referring the reader for brevity's sake to the above mentioned Manual of the Florida Department of Transportation) $U^{*}{ }_{c}$ is the critical friction velocity while $Z_{0}$ is a parameter calculated as a function of the critical Reynolds number $R_{e c}$. 
In actual fact, as the value of the $V / V_{c s}$ ratio in the experimental tests carried out has been estimated to be close to 1, Figure 3 reports for the various diameters tested (bearing in mind that greater scour occurs for values of $V=V_{c s}$ ) the envelope of the curves deduced by eqn. (3) with $V / V_{c s}=1$.

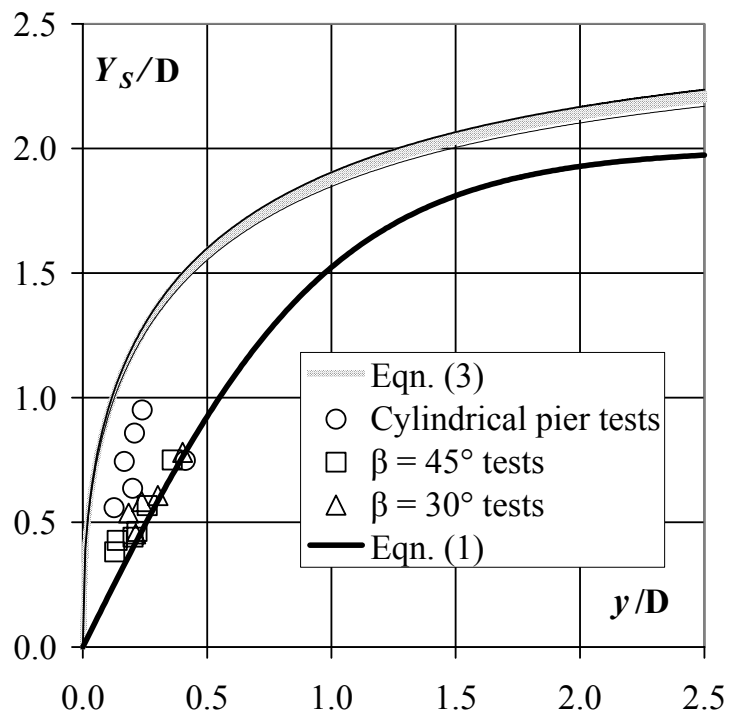

Figure 3: $\quad$ Comparison of the experimental values with equations (1) and (3).

The experimental values appear to be consistent with the values predicted by eqns. (1) and (3) and occupy an intermediate position between the curves represented in Figure 3.

In particular, the experimental values show that scour around piers with a truncated cone base is smaller and in good agreement with eqn. (1) proposed by Breusers, Nicollet and Shen [4]; this eqn. (1) seems, however, to underestimate the experimental values obtained for piers without a truncated cone base.

Furthermore, all the tests show scour values that are substantially lower than the estimate made using the eqn. (3) proposed by the Florida Department of Transportation [7].

Therefore, for the particular geometries used in the tests, eqn. (3) is a significantly precautionary envelope relation for estimating scour values.

\section{Conclusions}

The reduction in scour detected in the tests with the truncated cone base can be attributed to the modification of the complex distribution of velocity within the scour, which is characterized by components that are vertical and transverse to the flow direction, with fluid involved in vortex movements (horseshoe-vortex and wake-vortex). 
The pressure field deriving from the presence of the pier results in gradients that tend to concentrate whirling thread and then release them as dissipating vortexes that essentially cause localized erosion. On passing the pier, the wakevortex oscillates over time towards the sides: the non-uniform distribution of velocity then gives rise to vertical components.

The truncated cone foundation spatially modifies the whirling phenomena of the horseshoe-vortex (especially the counter-vortex) which in this case partly occur on solid surfaces; moreover the truncated cone accompanies the streamlines into the area where the horseshoe-vortex is shed: the different pattern of the hydrodynamic phenomenon on the one hand leads to a reduction in scour depth while, on the other, it creates a limited extension in the surface subject to erosion (which assumes an almost constant width for each value of $\beta$ ).

In actual fact, the modification of vortex phenomena is evidenced by the different position for the onset of erosion. Hager and Oliveto [10] pointed out that scour occurs around the pier in an area about $70^{\circ}$ from the channel axis (coinciding with the pier axis) and then extends on the whole front section where the horseshoe-vortex is located. In the experimental tests conducted with the truncated cone foundations it was observed that scour originates in an area close to an angle from the channel axis ranging between $70^{\circ}$ and $90^{\circ}$ for the various geometries before extending all over the pier front.

The preliminary tests of comparison and the results obtained with the particular foundation shape of the studied pier (which can also be adapted to more complex foundation geometries, i.e. like the ones reported in the Manual of the State of Florida) show a favourable shape_coefficient.

The obtained results suggest that the test schedule should be extended by including a greater number of diameters and varying the dynamic conditions of the stream flow, the height and angle $\beta$ of the truncated cone base, and the duration of the tests.

\section{Acknowledgements}

The present study is part of the research programme provided for in the National Interest Research Project (PRIN) 2005 - Naples Operative Unit.

\section{References}

[1] Laursen, E.M., Observation on the nature of scour, Proc., $5^{\text {th }}$ Hydraulic Conference, University of Iowa, Studies in Engineering, Iowa City (IA), 34, pp. 179-197, 1952.

[2] Laursen, E.M. \& Toch, A., Scour around bridge piers and abutments, Bulletin Iowa Highway Research Board, Ames (IA), No. 4, 1956.

[3] Melville, B.W., Local scour at bridge sites, University of Auckland, School of Engineering, Auckland (NZ), Report No. 117, 1975.

[4] Breusers, H.N.C., Nicollet, G. \& Shen, H.W., Local scour around cylindrical piers, Journal of Hydraulic Research, 15(3), pp. 211-252, 1977. 
[5] Hanco, S., Sur le calcul des affouillements locaux dans la zone des piles des ponts, Proc. 14th IAHR Congress, Paris (EU), 3, pp. 299-313, 1971.

[6] Sheppard, D.M., Final Report Scour at complex piers, Florida Department of Transportation Research Office, State of Florida, FDOT: BC354 RPWO 35, UF: 4910 45-04-799, 2003.

[7] Florida Department of Transportation, Bridge Scour Manual, State of Florida, http://www.dot.state.fl.us, 2005.

[8] Raudkivi, A.J. \& Ettema, R., Clear-water scour at cylindrical piers, Journal of Hydraulic Engineering ASCE, 109 (3), pp. 339-350, 1983.

[9] Melville, B.W. \& Raudkivi, A.J., Effects of foundation geometry on bridge pier scour, Journal of Hydraulic Engineering ASCE, 122(4), pp. 203-209, 1996.

[10] Hager, W.H. \& Oliveto, G., Further results to time-dependent local scour at bridge elements, Journal of Hydraulic Engineering ASCE, 131(2), pp. 97-105, 2005. 